\title{
电动汽车电接插件接触电阻振动特性的研究
}

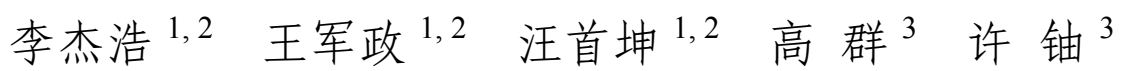 \\ (1. 北京理工大学复杂系统智能控制与决策国家重点实验室 北京 $100081 ;$ \\ 2. 北京理工大学伺服运动系统驱动与控制工信部重点实验室 北京 100081 ; \\ 3. 广东技术师范大学汽车与交通工程学院 广州 510635)
}

\begin{abstract}
摘要: 电动汽车市场发展迅速, 与此同时事故却频频发生, 其中很大一部分原因归处于电气设备的稳定性不足, 而电接插件 的安全性是电气设备稳定性的核心问题, 特别是针对高电压大电流的动力电池组。针对动力电池模组内部连接的电接插件展 开研究, 在目前电接插件制造工艺的基础上, 围绕振动工况下动力电池的电接插件的接触电阻以及接触性能一致性评估的核 心技术, 探索复杂振动工况下动力电池之间电接插件的接触电阻稳定性问题。首先构建面向振动工况下接触稳定性动力学模 型, 推导电接插件的振动与频率、幅值的关系。进一步地, 设计单体电池电连接器的电阻特性测试和电池模组的接触电阻振 动试验, 发现了接触电阻与接触面积、振动频率的变化规律。最后, 结合电动汽车对电源系统的安全需求及相关理论, 提出 一套高压接插件一致性评估思路, 指导电接插件性能在线评估以及结构优化, 为电动汽车高压电安全性提供了实际工程应用 和理论指导。
\end{abstract}

关键词: 电动汽车; 动力电池模组; 电接插件; 振动工况; 接触电阻

中图分类号: TM501

\section{Study on Contact Resistance Characteristics of Electrical Connectors of Power Battery Module}

\section{Jiehao $^{1,2}$ WANG Junzheng ${ }^{1,2}$ WANG Shoukun ${ }^{1,2}$ GAO Qun ${ }^{3}$ XU You ${ }^{3}$}

(1. Key Laboratory of Intelligent Control and Decision of Complex Systems,

Beijing Institute of Technology, Beijing 100081;

2. Key Laboratory of Servo Motion System Drive and Control, Beijing Institute of Technology, Beijing 100081;

3. School of Automotive and Transportation Engineering, Guangdong Polytechnic Normal

University, Guangzhou 510635)

\begin{abstract}
In recent years, the electric vehicle is developing rapidly, but the accident frequently occurred, which a large part of the reason is the insufficient stability of electrical equipment. However, the security of electrical connectors is the key to electrical equipment stability, especially for high voltage power battery packs. Based on the manufacturing process of electrical connectors, the high-voltage connectors of electrical contact are explored under the conditions of complicated working focusing on the three core technologies, including contact stress, contact resistance, and consistency evaluation. Firstly, the dynamic model of contact stability under vibration conditions is proposed, and the vibration relationship between the frequency and amplitude is studied. Furthermore, the vibration test of a single electrical connector and the battery module are carried out, which discover the contact resistance relationship between the contact area and the vibration frequency. Finally, in combination with the safety requirements and related theories of electric vehicles, an improved method of consistency evaluation for electrical connectors is proposed to guide the online performance evaluation and structure optimization and provides a viable test for improving the safety of high-voltage electric vehicles.
\end{abstract}

Key words: electric vehicle; electrical connector; contact resistance; vibration condition; consistency evaluatio

\footnotetext{
* 国家自然科学基金(61773060)、广东省科技计划项目应用型重大专项 (2015B010135006)和广东省青年创新人才 $(2017 K Q N C X 119)$ 资助项目。 20190628 收到初稿, 20200327 收到修改稿
} 


\section{0 前言}

近年来, 绿色能源推广应用得到高度重视, 电 动汽车行业正处在转型升级的黄金时期 ${ }^{[1]}$ 。其电气 设备的安全也备受关注, 尤其是动力电池模组上有 使用大量的电接插件，电接插件的安全性和稳定性 是电动汽车动力系统的核心问题。接插件的接触电 阻反映了接插件的电接触特性, 电接触点是实现导 通与分断电流功能的载体, 所以接插件的电接触特性 良好与否, 关系到整车的动力供给和管理系统的正常 运行 ${ }^{[2]}$ 。在实际工程中接插件的接触点之间由于接触面 的凹凸不平的原因, 正导电面积远小于实际面积, 产 生电流线弯曲并形成收缩电阻, 与触点表面的膜电阻 共同构成了接触电阻 ${ }^{[3]}$ 。电动汽车的电池管理系统需要 对电池包的电池状态保持监控 ${ }^{[4]}$, 满足可导通高电流、 大电压、使用寿命长和工作性能稳定的要求。为了实 现上述要求, 需要研究不同振动频率下动力电池接插 件的电接触特性, 以确保在安全规范规定振动频率下, 接插件能保持良好的接触特性。振动的存在, 会让电 池包的稳定性受到影响 ${ }^{[5]}$, 并且当外界冲击产生的振动 频率和电池包的固有频率接近时会引起共振, 导致机 械结构出现变形和受力不均, 从而引起接插件之间发 生较大位移影响其电接触特性。

与工业用途的电接插件相比, 动力电池模组的 电接插件除了考虑接触电阻的情况 ${ }^{[6]}$, 同时还要考 虑各种复杂工况, 包括空间环境、振动工况、温度 影响等对电接插件的影响, 以及产品出厂和长时间 使用后的一致性问题 ${ }^{[7-10]}$ 。其中复杂振动工况对电 接插件接触特性的影响以及电接插件一致性问题是 决定电接插件能够安全使用的关键因素。

在振动工况下, 接插件的电接触特性与本身接 触应力、振动频率、振动方向等相关。周航 ${ }^{[11]}$ 通过 搭建了基于自混合干涉效应的微位移测量实验平 台, 实现电接插件微振动的检测及损伤的识别, 但 未涉及损伤程度与固有频率之间关系的研究。周权 等 ${ }^{[12]}$ 针对接触件进行了力学分析, 建立了接触件单 脚分离力、接触电阻与结构参数之间的关系, 但未 建立接触电阻和接触件分离力的约束关系。郑强 ${ }^{[13]}$ 研究了电接插件在环境温度、湿度和振动应力综合 作用下的加速寿命试验方案的优化设计模型, 并对 电接插件的失效机理进行了探讨, 得出电接插件的 接触寿命模型, 但未考虑各种湿度情况下对电接插 件可靠性的研究。潘骏等 ${ }^{[14]}$ 提出了一种接触件接触 面积的迭代计算方法, 并对接触件的接触情况进行
了运动仿真, 得到了接插件拔插过程中接触压力、拔 插力的变化情况和应力分布情况, 并通过实验验证理 论的有效性, 但是未考虑接触压力, 拔插力和接触电 阻三者之间的关系。靳方建 ${ }^{[15]}$ 结合电接触理论, 建立 了电接插件接触电阻模型, 并确定了接触压力与接触 电阻之间的关系，但未考虑长期工作过程对接触件应 力松弛或蠕变等现象的影响。对于电动汽车电接插件, 其使用工况复杂多变, 在长时间的复杂振动下, 电接 插件容易发生松动获得接触应力减小的情况, 这种现 象会导致接触电阻变大，甚至接触不良导致电弧的产 生。钟泽南对汽车连接器进行了仿真分析, 得出当振 动频率超过某一上限, 连接器的接触压力会大幅度波 动甚至导致连接器脱离。因此, 开展振动工况下电动 汽车动力电池模组的连接器接触电阻的变化特性研究 是汽车安全保障中非常重要的环节 ${ }^{[16-17]}$ 。

综上分析, 动力电池模组的电接插件在电动汽 车实际工程应用中存在以下几个突出的问题。

(1) 为保证电接触应力以及稳定性, 工程上通 过增加接插件面积的方法, 保证产品达到相应电接 触指标。采用弹片机构、自锁机构等固定装置设计 方式，导致产品体积大且轻量化程度不高。

（2）设计了比较完备的安全机构, 内部添加接 触故障检测系统，保证电接触的合理性。考虑短路 保护，在接触件内部设置熔断机构，保证在过流情 况下接触隔离。考虑防水等级, 增大防水胶垫厚度 及面积, 保证系统防水性能。但是结构复杂, 而且 针对不同需求的电接插件, 必须单独开发模具, 造 成电接插件设计、生产的兼容性低, 开发成本大。

（3）电接插件产品在生产、安装过程中缺乏系 统的接触一致性检测。在实际工程上主要采用抽样 抗压、抽样抗拉等破坏性试验, 缺乏系列产品的全 功能检测手段, 这也导致接插件的稳定性不高。

本文的目的是在目前电接插件制造工艺的基础 上, 针对电动汽车动力电池模组内部的接插件安全 性问题展开研究，探索振动工况下动力电池之间电 接插件不同链接方式的电阻变化规律，建立振动工 况下电接插件的接触稳定性动力学模型及接触电阻 变化模型, 并结合电动汽车对电气设备的安全需求 及相关理论, 提出一套电接插件一致性评估思路, 指导电接插件性能在线评估以及结构优化，提高动 力电池模组接插件的安全性。

\section{1 接插件振动特性动力学分析}

为了更好地描述电接插件在振动工况下接触电 
阻的变化规律, 本文在自主研发的管柱型结构单元 电池包的基础上, 展开对动力电池模组电接插件的 研究, 如图 1 所示。电接插件通过点焊的方式连接 在 32650 磷酸铁锂电池的一极, 电接插件另一端有 螺纹结构, 可以让锂电池进行串联连接。同时接插 件上有 4 个 $\mathrm{U}$ 型弹片, 构成了插片-插孔的连接方式, 可以把串联好的单管电池组, 根据功能需求进行并 联, 构成电动汽车的动力电池包。同时电压传感器, 温度传感器等装置都会布置在接插件的周围, 与所 研发的管柱型结构单元电池拓扑结构, 行成一套完整 的动力电池包。由于电池包的锂电池在相对封闭的环 境下工作, 电接插件的安全性和稳定性成为了电池包 的核心关键部件, 而本文所研究的目的在于找出高压 动力电池组的接插件的电阻变化特性。进一步地为高 安全性、轻量化的电动汽车动力电池包奠定基础。

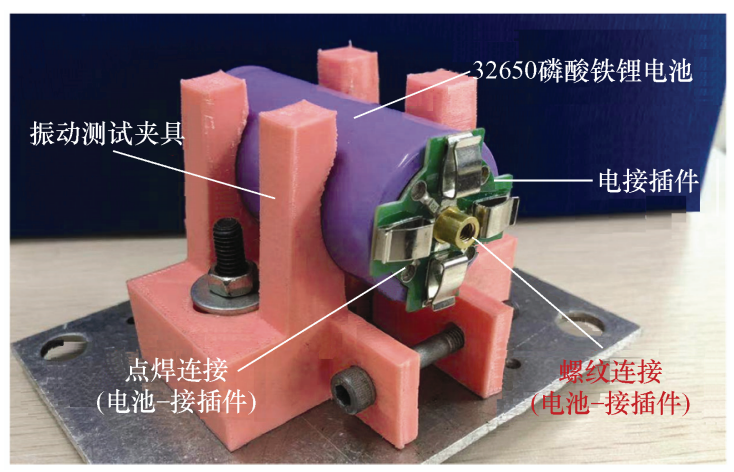

图 1 电动汽车动力电池组电接插件实物图

本文所研究的电源系统电接插件简化动力学模 型如图 2 所示。在对动力电池的电接插件进行动力 学推导的过程中, 考虑接插件的振动电阻特性, 因

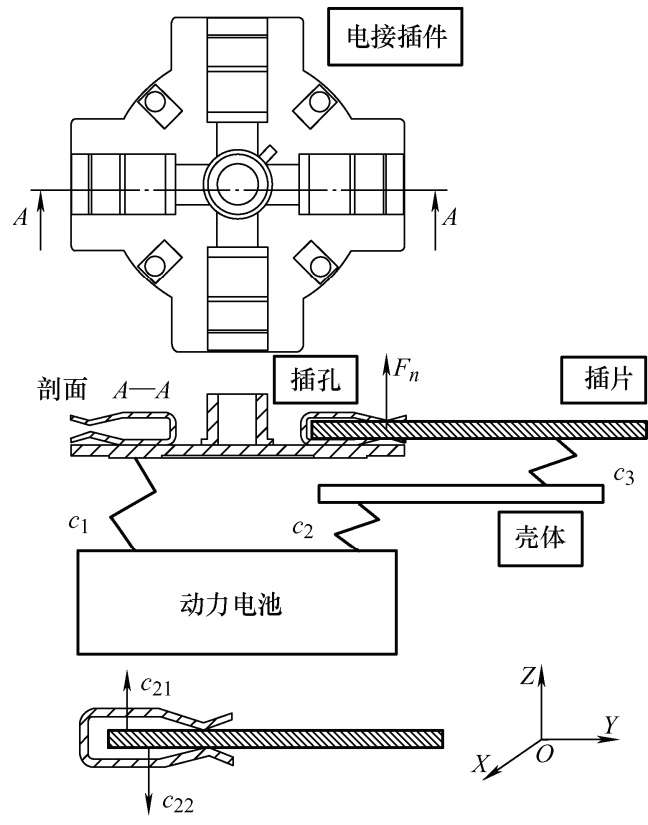

图 2 电接插件简化动力学模型
此可以把这个问题看成是经典的弹性体振动。而接 插件的接触方式为插针接触和插片接触, 可以转化 为粱的弯曲振动这一典型问题解决 ${ }^{[18-19]}$ 。由于插片 和插针的简化模型等效, 因此以接插件的配合关系 插孔-插片的方式分析电接触振动特性。电接插件与 动力电池通过电焊的方式连接, 设 $c_{1}$ 和 $c_{3}$ 均视为刚 性连接, 接触压力为 $F_{N}$, 螺纹连接 $c_{2}$ 等效为上下 $c_{21} 、 c_{22}$ 的连接, 插头的质量是 $M$ 。电动振动台与接 插件之间通过机械夹具刚性连接, 接插件的振动与 振动台提供的激振近似相同。

首先设定电接插件在 Stewart 六自由度振动测 试平台下做稳定的正弦波振动, 其角速度为 $\omega$ 和振 幅为 $A$, 则接插件之间所产生的振动距离为

$$
h(t)=A \sin \omega t
$$

根据达朗贝尔方程, 可以得到电接插件的动力 学方程为

$$
M \frac{\mathrm{d}^{2} h(t)}{\mathrm{d} t^{2}}=\left(-c_{21}-c_{22}\right)[\dot{h}(t)-A \sin \omega t]
$$

结合图 2 所示的动力学模型, 上式可以转化为

$$
\frac{\mathrm{d}^{2} h(t)}{\mathrm{d} t^{2}}+\frac{c_{21}+c_{22}}{M} h(t)=\frac{c_{21}+c_{22}}{M} A \sin \omega t
$$

进一步地, 考虑初始条件为静止状态, 即位移 和初速度为零, 设

$$
\eta^{2}=\frac{c_{21}+c_{22}}{M}
$$

则有

$$
\frac{\mathrm{d}^{2} h(t)}{\mathrm{d} t^{2}}+\eta^{2} h(t)=\eta^{2} A \sin \omega t
$$

方程的通解为

$$
h(t)=B_{1} \cos \eta t+B_{2} \cos t+\frac{\eta^{2}}{\eta^{2}-\omega^{2}} A \sin \omega t
$$

由于接插件的电阻振动特性可以转化为粱的 弯曲振动问题, 看成经典的弹性体振动。由材料 力学的简化粱理论 ${ }^{[20]}$, 插片-插孔具有相同的横截 面, 忽略剪切变形和转动惯量的影响, 且插片的 长度远大于其高度, 因此可以把电接插件等效为 满足欧拉-伯努利方程的简化粱。结合振动理论所 给出的粱的弯曲振动关系 ${ }^{[19,25]}$, 可以得出接插件 的振动函数为

$$
\begin{aligned}
& h(x, t)=\left(e^{\gamma x}+e^{-\gamma x}-2 \cos \gamma x\right) \frac{\varphi \omega^{2} A}{\eta^{2}-\omega^{2}} \times \\
& \frac{1}{\sqrt{\varphi^{2}+\left(E I \omega^{3}\right)^{2}}} \sin \left(\omega t+\arctan \frac{E I \omega^{3}}{k}\right)
\end{aligned}
$$

其中, 振动函数是一个与位置 $x$ 有关的常数 


$$
X(x)=e^{\gamma x}+e^{-\gamma x}-2 \cos \gamma x
$$

时域函数为

$$
\begin{aligned}
& Y(t)=\frac{\varphi \omega^{2} A}{\eta^{2}-\omega^{2}} \frac{1}{\sqrt{\varphi^{2}+\left(E I \omega^{3}\right)^{2}}} \times \\
& \sin \left(\omega t+\arctan \frac{E I \omega^{3}}{k}\right)
\end{aligned}
$$

该方程表示了电接插件在接插时间 $t$ 的振动与 频率、振幅的关系。

\section{2 振动工况下接插件电接触试验}

接插件的接触特性关系到动力电池系统的工作 稳定性, 根据不同的振动工况, 接插件的接触电阻 会受到振动频率的影响 ${ }^{[21]}$ 。在电接触动力学动态模 型的基础上, 分析振动频率、振动时间与接触电阻 变化规律, 并以此规范动力电池模组的电连接器结 构, 从而促进电连接器的轻量化和高安全性。

振动试验平台如图 3 所示, 主要由六自由度振 动测试台、直流低电阻测试仪、示波器、动力电 池电接插件和数据采集装置组成。需要注意的 是, 本文所提及的电接插件主要针对的是与锂电 池之间的连接器件, 是安装在自主研发的管柱型 结构单元动力电池包的核心零部件。试验测试分 为两部分内容, 一是单体电池接插件电接触特性 的振动测试, 选取两种典型的插拔类型, 分别是 插针-插孔方式和插片-插孔方式; 另一个是动力 电池包接插件的动态测试。

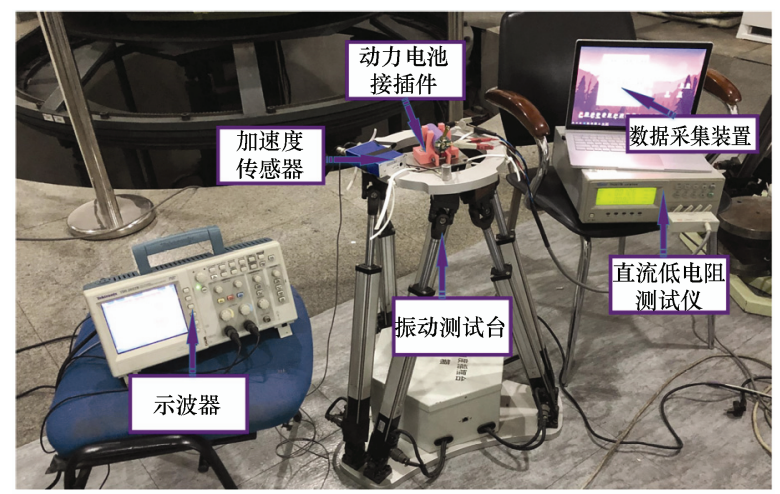

图 3 电接插件振动试验平台

振动试验整体思路: 由电接插件的振动动力学 关系得出振幅与频率, 振动时间的函数关系。电接 插件的主要功能是连接电池与电池之间, 电池组与 电池组之间, 根据功能需求组成不同类型的电池包 结构。基于此, 设计了插针和插片两种连接方式的 电阻振动测试。研究在振动工况下, 电接插件的电
阻变化特性和标准差分布情况。同时, 考虑电池包内 部的接插件稳定性, 设计了电池包电接插件的电阻振 动测试。最后通过数据收集和电阻特性分析, 总结在 振动工况下，电接插件的电阻变化规律。

具体的振动模式下电接插件的电阻振动实验步 骤如下所示。

(1) 将接插件与电池的单体模块安装到测试夹 具上, 并固定在振动测试台, 连接好数据采集电路。

(2) 针对动力电池模组的电连接器振动测试, 选取一个模组, 由 $3 \times 3 \times 10$ 阵列组成, 每个并联模 块设计为 $A, B, C, D, E, F$ 六个采集面, 测量各 个接插件的接触电阻变化情况。

(3) 通过上位机的控制软件，调节振动测试台 的振动频率进行试验测试。采用直流低电阻测试仪 对接插件在不同振动工况下的电阻变化进行测量。

(4) 收集试验数据, 分析与归纳接插件的接触 电阻振动特性。

\section{3 试验结果及分析}

\section{1 单体接插件的电接触振动特性}

为了直观地表现出在不同振动频率的动力电池 电接插件的接触特性，本文针对自主研发的管柱型 结构化单元动力电池模组的电接插件连接方式的动 态电阻特性展开研究, 选取不同振动频率下的接触 电阻平均值反映振动特性的稳定性，同时通过不同 振动频率下接触电阻的标准偏差来反映其接触特性 的一致性。

本文选择了课题组自主研发的管柱型结构 化单元动力电池模组内部的电接插件, 连接方式 分别是典型的插针-插孔方式和插片-插孔方式, 如图 4 所示。通过不同振动工况下的接触电阻振 动试验, 分析与归纳不同振动频率下接插件电阻 变化规律 ${ }^{[22]}$ 。

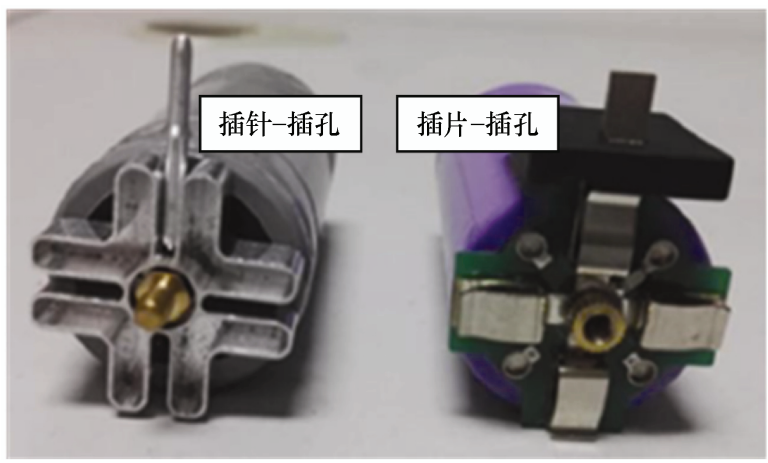

图 4 两种典型的电接插件

图 5 所示的是两种接触类型接插件在不同振动 
频率下接触电阻变化规律。可以看出, 插针配合的 接插件在振动工况下, 接触电阻远远大于插片配合 方式, 这说明了插片的配合方式受振动频率的影响 较小, 稳定性较高。值得注意的是, 振动频率在 $40 \mathrm{~Hz}$ 以前, 接触电阻变化波动较大, 接触特性变 差, 波动的趋势均为先增大后减小。在这个阶段, 插针的配合方式会出现急剧变化的阶段, 但插片方 式的变化相对缓和, 这说明了在接触电阻波动的阶 段, 插片配合的接插件的稳定性较好。在 $40 \mathrm{~Hz}$ 以 后, 接触电阻变化波动极小并趋于稳定, 且不受振 动频率的影响, 接插件的稳定性较高。

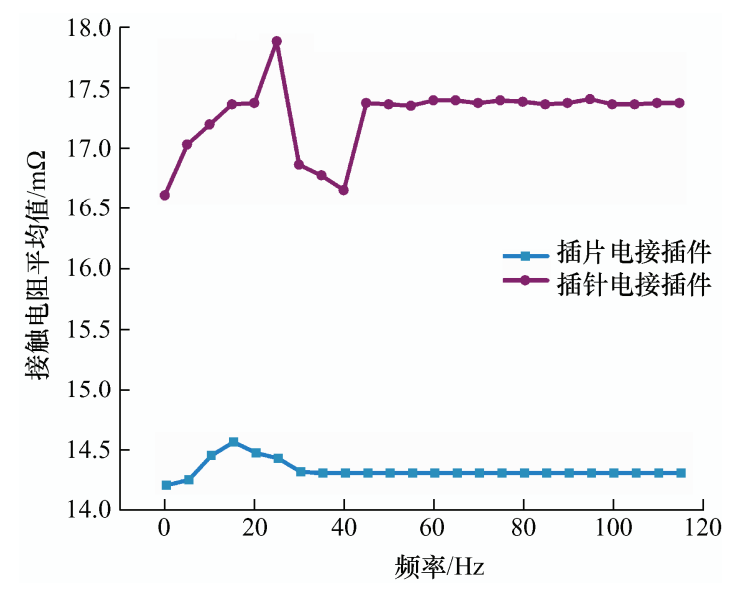

图 5 插针-插片接插件接触电阻变化特性

进一步研究两款接插件的接触电阻标准偏差的 变化规律, 如图 6 所示, 揭示了在不同的振动频率 下, 接插件的接触电阻稳定性。从接触电阻的标准 偏差曲线趋势可以明显看出, 插片的配合方式稳定 性要插针的配合方式更可靠。在 $40 \mathrm{~Hz}$ 以前, 插针 稳定性的受振动频率的影响较大, 数据浮动快。

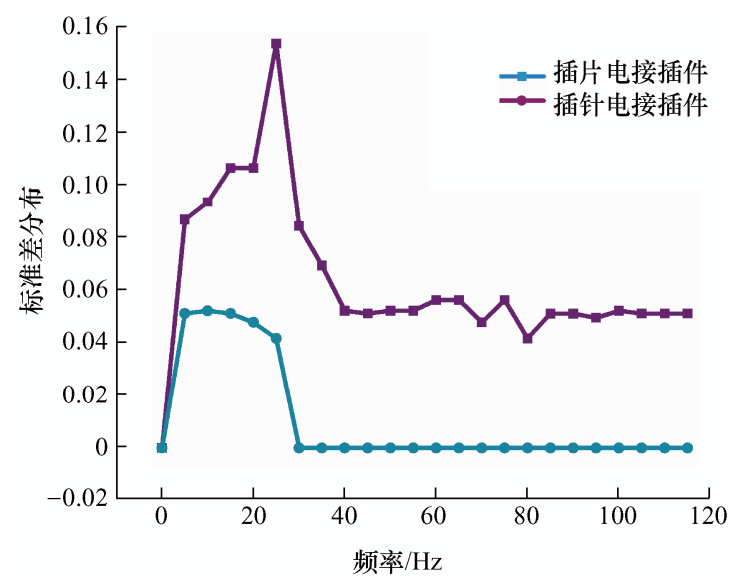

图 6 插针-插片接插件接接触电阻标准差变化特性

通过分析上述两款不同的配合方式的接插件振 动试验结果, 结合接触电阻在不同频率下的平均值 和标准偏差变化规律, 可以看出插片配合方式的接
插件稳定性更加可靠。同时, 插片的方式在振动频 率 $40 \mathrm{~Hz}$ 以后, 接触电阻就不会再变化, 而且插针 的方式在频率 $50 \mathrm{~Hz}$ 以后也有同样的结果, 而且这 个结果与后期的电池包接插件的振动测试与之呼 应。这说明了在机械结构和动态影响下, 接插件会 有一个稳定区域。这说明了增大接触面积, 使得接 插件的接触电阻减小, 振动频率对接插件的接触特 性影响较小。因此, 可以推出接触电阻与振动频率 呈负反馈变化规律。

\section{2 接插件接触电阻振动曲线基本算法}

通过上述接插件的振动试验, 与测量值直接 相关的是实验过程中的振动频率, 进一步推导出 接触电阻与振动频率的数学关系, 以解释实验记 录的离散的数据点。接触电阻曲线的拟合算法的 具体过程为: 首先初始化程序, 以 $a$ 为起点, $b$ 为 间隔, $c$ 为终点。计算多项式的值 $y_{i}=\operatorname{ployval}\left(\Psi, x_{i}\right)$, 并计算响应函数的系数 $\rho=$ ployfit $(x, y, n)$ 和 $y_{e}=$ $\operatorname{ployval}\left(\Psi, x_{i}\right)$, 最后求出接触电阻 $R=1-\operatorname{sum}\left(\left(y_{i}-\right.\right.$ $\left.\left.y_{e}\right)^{2}\right) / \operatorname{sum}\left(\left(y_{i}-\operatorname{mean}(y)^{2}\right)\right)$ 。

为了更好地表达电阻测量值与振动频率的内在 关系, 通过 Matlab 进行对振动频率为自变量, 电阻 值为因变量进行拟合, 求解出函数关系式。设有实 验数据 $\left(x_{i}, y_{i}\right), i=1,2, \cdots, n$, 设电阻函数为 $F(\Psi, x)$ 在 点 $x_{i}$ 处的函数值与观测数据偏差的平方和达到最小 使得

$$
\min \sum_{i=1}^{n}\left[F\left(\Psi, x_{i}\right)-y_{i}\right]^{2}
$$

最终得到最适合的接触电阻与振动频率的变化 规律表达式为

$$
\Gamma_{R}=F(f, t)=A f^{4}+B f^{3}+C f^{2}+D f+E
$$

式中, 接触电阻为 $\Gamma_{R}$, 振动频率为 $f$ 。

\section{3 电池模组的接插件振动特性分析}

动力电池包是电动汽车的核心部件, 其性能 直接影响着安全性和稳定性, 而电池模组内部接 插件是核心零部件, 直接决定着电池包的安全 性。汽车在行驶过程中会受到不同程度的振动, 而振动会直接影响到电池包的安全性。振动的存 在, 会影响电池模组的接插件接触电阻的大小, 接触不良会导致电路短路, 容易造成电池包起 火, 从而影响到续航里程和寿命, 对车辆安全构 成严重威胁。

因此, 为了更好地描述电池模组内部的接插件 接触电阻与振动频率的关系 ${ }^{[23]}$, 对管柱型结构化单 元的动力电池包进行振动测试, 具体的试验步骤如 图 7 所示。首先, 选取一个动力电池模组, 其电池 
拓扑结构由 $3 \times 3 \times 10$ 阵列组成。然后, 划分每个 并联模块设计为 $A, B, C, D, E, F$ 六个采集面, 测量各个接插件在静止状态下的接触电阻变化
情况。接着设定响应的振动频率, 对电池模组进 行振动测试, 并记录振动前后的各个采集面的接 触电阻变化过程。
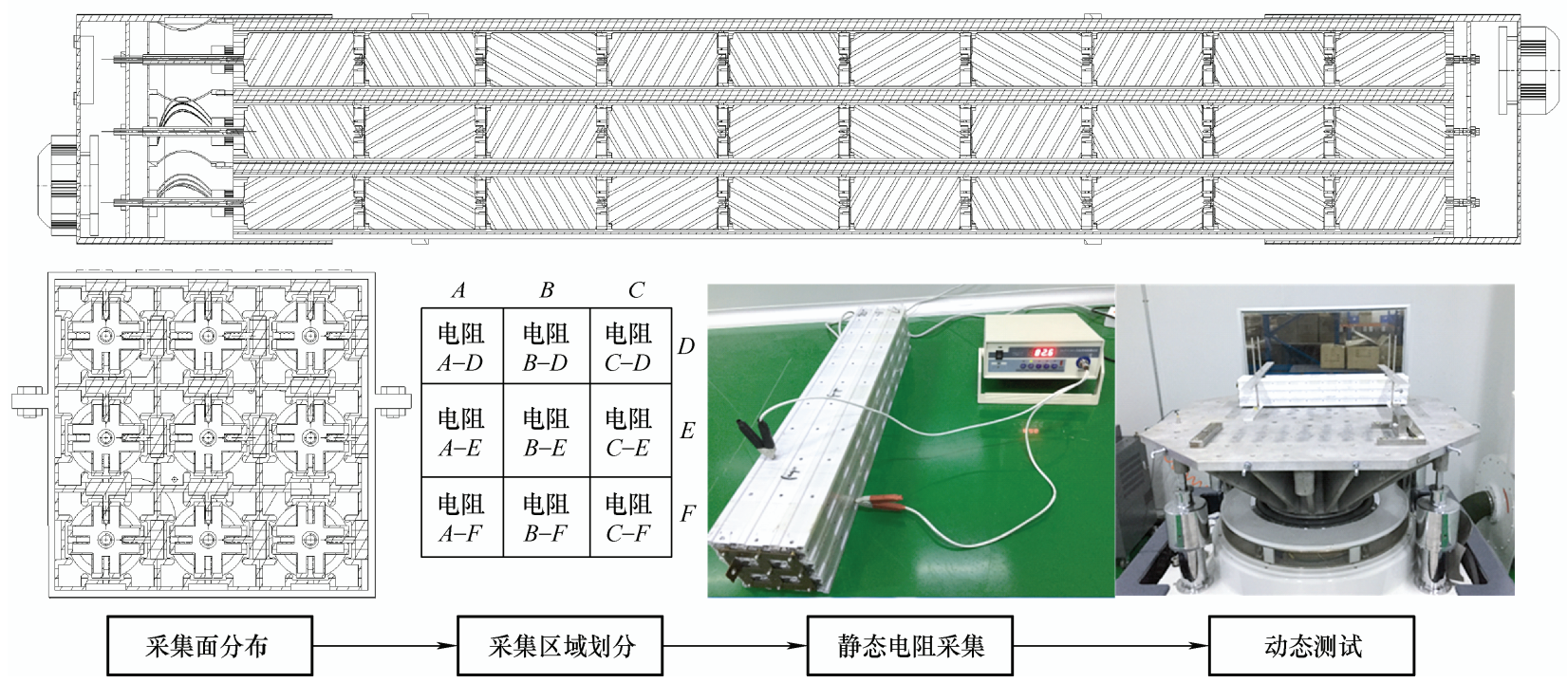

图 7 动力电池模组试验测试

为了能够配合振动平台, 本课题组自主设计了 电池包振动测试夹具 ${ }^{[24]}$ 。该夹具由四根长度为 $700 \mathrm{~mm}$, 宽度为 $40 \mathrm{~mm}$, 厚度为 $10 \mathrm{~mm}$ 的带有若 干间距相等通孔的固定条, 八根用于夹紧电池模组 及振动台的螺杆。固定端上通孔一面为圆形设计, 可满足螺杆通过, 另一面设置内六角凹槽, 可满足 螺杆外六角头的放置及固定。

动力电池模组电连接器的一致性振动测试使用 的机器为 ES-50-445 型振动台, 本振动参数设置为 初始振动频率为 $10 \mathrm{~Hz}$, 固定位移量为 $15 \mathrm{~mm}$, 固 定加速度为 $1 \mathrm{~m} / \mathrm{s}^{2}$, 振动测试时间为 $60 \mathrm{~min}$ 。本振 动测试过程中电池模组通过橡胶垫片进行绝缘隔 离, 防止短路等问题对振动测试造成的影响。通过 一致性振动测试, 电池模组的接插件接触电阻振动 前后试验结果如图 8 所示。

在动力电池模组内部振动前后的电接插件电阻 阻值变化的三维空间分布图, 明显可以看到振动后 电池模组对应的接触电阻普遍降低了, 只有极少数 接触口电阻变大。进一步分析接插件的动态电阻降 低的原因, 考虑到电池与电池之间的连接块为铝制 品, 而金属电阻会随着温度的升高而变大, 但是铝 的导热性能好, 电池模组有散热用的分散以及通风 口, 因此电池之间的温度对金属电阻影响较低。在 不同的振动频率下, 其接触电阻会有所不同, 由于 间隙的随机性, 振动可能导致连接块的接触面积变 大或者变小从而改变电阻的大小。从振动试验结果可 以看到振动后的电阻变小, 振动迫使电接插件之间摩

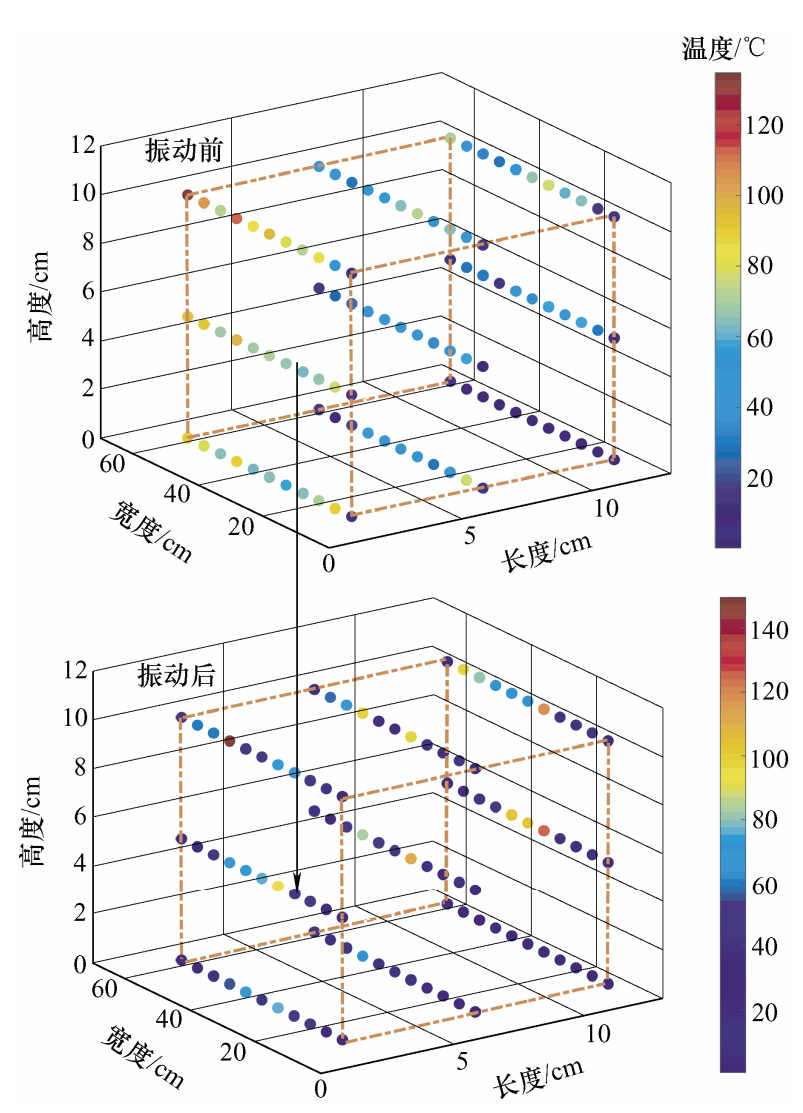

图 8 电池模组接插件振动测试电阻三维分布图

擦增大, 导致接触面积增大, 而小部分连接块间由于 振动导致接触错位从而使接触面积变小, 电阻变大。 从振动试验表象看, 温度和接触面积是导致电阻的变 化的因素, 由于电池包散热系统的设计, 在振动过程 中接触间隙是电接插件的接触电阻变化的主要因素。 
因此, 可以发现电池模组振动试验结果, 与 单个接插件的振动试验结论一致。在电池输出的 电压一致时, 由于接触面积的不同导致了电阻的 不一致性, 使得电接插件的电流也会不同。电流 的不一致性会导致接插件的温度不同。当聚集到 接插件的温度增加过快, 短时间内聚集在电池模 组内部的热量会急速增加, 进而导致电源系统存 在安全隐患。

\section{4 电接插件一致性评估思路}

振动会对接插件的用于导电的接触表面造成影 响, 随着时间延长, 结构产生松动, 其接触面积会 产生波动。根据上述振动工况下接触电阻变化情况 的是试验数据, 基于电接触理论, 通过数学分析和 统计的方法可得到接触电阻 $R$ 与频率 $f$ 和振动作用 时间 $t$ 的函数关系为 $R=F(f, t)$ 。同时, 在未来工作 中, 进一步研究电接插件力学指标, 连接强度和接 触面积, 同时考虑抗压程度、拉伸程度等。因此, 综合考虑电接插件的各项力学性能, 结合接触电阻 变化模型, 进一步提出一致性评估思路。假设电接 插件的各项力学性能指标为 $\tau_{1}, \tau_{2}, \cdots, \tau_{n}$, 各项力学 指标的重要性为 $\xi_{1}, \xi_{2}, \cdots, \xi_{n}$, 则力学稳定性模型: $\Gamma_{E}=\tau_{1} \xi_{1}+\tau_{2} \xi_{2}+\cdots+\tau_{n} \xi_{n}$ 。综上分析, 电接插件一 致性评价函数为

$$
F=\Gamma_{R} \frac{1}{R}+\Gamma_{E} E
$$

\section{4 结论}

本文针对电动汽车动力电源系统的接插件接触 电阻振动特性进行了深入的研究, 探究在振动工况 下的动力电池电接插件的电阻变化, 揭示了接插件 的接触电阻与振动频率、幅值的数学关系。同时, 考虑了 “插孔-插针” 和 “插孔-插片” 这两种工程 应用最广泛的接触方式进行试验测试, 发现了电接 插件在单体电池和电池模组的接触电阻与接触面 积、振动频率的变化规律。根据电接插件的接触电 阻模型和力学稳定性指标, 进一步地提出了电接插 件的一致性评估函数, 指导电接插件性能在线评估 以及结构优化, 提高了电源系统的安全性, 为电动 汽车电接插件的维护和评估提供了实际工程应用 和理论指导。

在未来的研究工作, 为了更全面地评估和测试 高安全性的动力电池连接件, 将深入研究复杂振动 工况下电连接器的稳定性力学理论研究及应用, 包 括建立电连接器仿真分析模型, 分析不同振动方向 与频率、不同连接结构的电连接器接触对间的应力
应变情况, 同时考虑建立接触压力、插拔力接触件 与结构参数之间的关系。与此同时, 面向高接触面 积、多芯兼容的高稳定性电连接器结构优化设计进 行研究, 尝试在电连接器螺丝和垫片添加卡位结构, 构建一套基于卡位电连接器的摩擦因数模型以及电 阻模型, 以此指导电连接器结构优化, 提高其连接 稳定性, 并对电连接器接触一致性评估方法进行理 论研究及实际工程应用。

\section{参 考 文 献}

[1] 李晋川. 电连接器接触电阻测试方法探讨 [J]. 机电元 件, 2014, 34(6): 41-44.

LI Jinchuan. Discussion on test method of contact resistance of electrical connector[J]. Electromechanical Components, 2014, 34(6): 41-44.

[2] 黄剑飞, 张海宝, 李华武. 铜电解极板与导电排的接触 电阻探析 [J]. 有色冶金设计与研究, 2016, 37(1): 21-22. HUANG Jianfei, ZHANG Haibao, LI Huawu. Analysis on contact resistance of copper electrolysis electrode plate and conducting bar[J]. Nonferrous Metals Engineering and Research, 2016, 37(1): 21-22.

[3] XU Liangjun, LU Na, LIN Xueyan, et al. Electrical contact theory, application and technology[M]. Beijing: Mechanical Industry Press, 2010.

[4] 吴楠, 庄劲武, 江壮贤, 等. 螺栓紧固铜排间接触电阻 研究[J]. 海军工程大学学报, 2016, 28(6): 28-31.

WU Nan, ZHUANG Jinwu, JIANG Zhuangxian, et al. Contact resistance between bolt fastening copper platoons[J]. Journal of Naval University of Engineering, 2016, 28(6): 28-31.

[5] 柏小平, 李国伟, 翁桅, 等. 电触头表面状态对接触电 阻的影响和改善方法 [J]. 电工材料, 2013, 1(1): 10-15. BAI Xiaoping, LI Guowei, WENG Wei, et al. Effects of contact surface condition on contact resistance and improving methods $[\mathrm{J}]$. Electrical Engineering Materials, 2013, 1(1): 10-15.

[6] NIV A, GHARGHI M, GLADDEN C, et al. Near-field electromagnetic theory for thin solar cells[J]. Physical Review Letters, 2012, 109(13): 138701.

[7] KUMAR R, OSHIMA T, MIKAMI S, et al. Damage identification in a lightly reinforced concrete beam based on changes in the power spectral density[J]. Structure and Infrastructure Engineering, 2012, 8(8): 715-727.

[8] CHEN Wenhua, GAO Liang, LIU Juan, et al. Optimal design of multiple stress accelerated life test plan on the 
non-rectangle test region[J]. Chinese Journal of Mechanical Engineering, 2012, 25(6): 1231-1237.

[9] GAO Liang, CHEN Wenhua, LIU Juan, et al. Design criteria for planning multiple stresses accelerated life test[C]// The Proceedings of 2011 9th International Conference on Reliability Maintainability \& Safety. IEEE, 2011: 1141-1146.

[10] CHEN Wenhua, LIU Juan, GAO Liang, et al. Accelerated degradation reliability modeling and test data statistical analysis of aerospace electrical connector[J]. Chinese Journal of Mechanical Engineering, 2011, 24(6) : 957-962.

[11] 周航. 电接触构件微振动的检测与损伤识别研究 [D]. 秦皇岛: 燕山大学, 2015 .

ZHOU Hang. Micro vibration testing and damage identification on sliding electrical contact component[D]. Qinhuangdao: Yanshan University, 2015.

[12] 周权, 雷永涛, 叶宇, 等. 电连接器冠簧接触件结 构分析及其接触可靠性 $[\mathrm{J}]$. 机电元件, 2015, 35(1): 44-47.

ZHOU Quan, LEI Yongtao, YE Yu, et al. Structural analysis and contact reliability of crown spring contact of electrical connector[J]. Electromechanical Components, 2015, 35(1): 44-47.

[13] 郑强. 电连接器环境应力可靠性试验统计分析[D]. 上 海: 上海交通大学, 2010 .

ZHENG Qiang. Statistical analysis of environmental stress reliability test of electrical connectors[D]. Shanghai: Shanghai Jiao Tong University, 2010.

[14] 潘骏, 靳方建, 陈文华, 等. 电连接器接触件结构分析 与插拔试验 $[\mathrm{J}]$. 中国机械工程, 2013,24(12): 1636-1641. PAN Jun, JIN Fangjian, CHEN Wenhua, et al. Structural analysis of electrical connector contacts and insertion extraction test[J]. China Mechanical Engineering, 2013, 24(12): 1636-1641.

[15] 靳方建. 电连接器接触件可靠性分析与高温插拔试验 [D]. 杭州: 浙江理工大学, 2012.

JIN Fangjian. Reliability analysis and high temperature plug-in test of electrical connector contact[D]. Hangzhou: Zhejiang Sci-Tech University, 2012.

[16] 钟泽南. 汽车连接器的动态性能分析[D]. 北京: 北京邮 电大学, 2015 .

ZHONG Zenan. Dynamic performance analysis of automotive connectors[D]. Beijing: Beijing University of Posts and Telecommunications, 2015.
[17] TAN Zhongqiang, ZHAO, Mingyan, WANG Shijiao. Analysis of mechanism reliability in anti-dislocation locking of space cable-strut deployable articulated mast[J]. Advanced Materials Research, 2013, 753(1): 1145-1150.

[18] 支宏旭. 综合环境应力下航空电连接器电接触特性的 实验研究[D]. 哈尔滨: 哈尔滨工业大学, 2015.

ZHI Hongxu. Experimental study on electrical contact characteristics of avionics connectors under integrated environmental stress[D]. Harbin: Harbin Institute of Technology, 2015.

[19] 方同, 薛璞. 振动理论及应用 $[\mathrm{M}]$. 西安: 西北工业大 学出版社, 1998.

FANG Tong, XUE Pu. Vibration theory and application[M]. Xi'an: Northwestern Polytechnical University Press, 1998.

[20] 王世娇, 陈文华, 钱萍, 等. 航天电连接器的可靠性设 计建模[J]. 机械工程学报, 2017，53(10): 180-186.

WANG Shijiao, CHEN Wenhua, QIAN Ping, et al. Relia bility design model of aerospace electrical connector[J]. Journal of Mechanical Engineering, 2017, 53(10): 180-186.

[21] CHEN Wenhua, LIU Juan, GAO Liang, et al. Step-stress accelerated degradation test modeling and statistical analysis methods $[\mathrm{J}]$. Chinese Journal of Mechanical Engineering, 2013, 26(6): 1154-1159.

[22] 杜永英, 孙志礼, 吕春梅, 等. 基于 ANSYS 某型电连 接器插拔过程的可靠性分析 [J]. 中国工程机械学报, 2015, 13(6): 545-549.

DU Yongying, SUN Zhili, LÜ Chunmei, et al. Reliability analysis on terminal contact parts for electrical connector based on ANSYS[J]. Chinese Journal of Construction Machinery, 2015, 13(6): 545-549.

[23] 李艳生, 杨美美, 芦娜, 等. 同心连接器的力学性能测 试技术和系统研究 [J]. 仪器仪表学报, 2017, 38(2): 328-335.

LI Yansheng, YANG Meimei, LU Na, et al. Research on testing technology and system of mechanical properties for a concentric connector[J]. Chinese Journal of Scientific Instrument, 2017，38(2): 328-335.

[24] 黄超, 许铀, 高群, 等. 一种电池包振动测试夹具: 中 国, 201620850605.4 [P].2017-03-15.

HUANG Chao, XU You, GAO Qun, et al. A test equipment of battery pack vibration: China, 201620850605.4[P], 2017-03-15. 
[25] 任万滨, 崔黎, 翟国富, 等. 电连接器接触件插拔特性 与接触电阻的仿真分析 $[J]$. 机电元件, 2012, 32(3): 40-44.

REN Wanbin, CUI Li, ZHAI Guofu, et al. Simulation of contacts inserted characteristics and contact resistance for electrical connector[J]. Electromechanical Components,
2012, 32(3): 40-44.

作者简介: 李杰浩(通信作者), 男, 1989 年出生, 博士研究生。主要研 究方向为动力电源系统及机器人智能控制。

E-mail: jiehao.li@bit.edu.cn

王军政, 男, 1964 年出生, 博士, 教授, 博士研究生导师。主要研究方 向为伺服运动驱动与控制、机器人控制和负载模拟与静动态试验。

E-mail: wangjz@bit.edu.cn

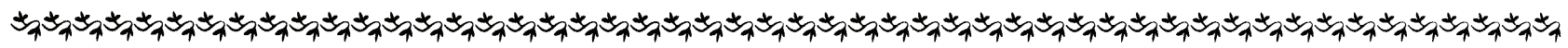

第 9 届上银优秀机械博士论文奖一一佳作奖

\section{$\mathbf{V}$ 形直线超声电机动力学建模与应用研究}

作 者: 李晓牛

毕业学校: 南京航空航天大学

指导教师：赵淳生、姚志远

$\mathrm{V}$ 形直线超声电机具有输出力大、运行速度高和行程长等优点, 在精密工程、航空航天和生物工程等领域展现广阔的应 用前景。目前缺乏其动力学建模、分析和控制的研究，难以满足结构优化、性能预测和驱动控制系统设计的需求，限制了该 类电机性能的提升, 制约了电机的应用。本文的主要研究内容和成果如下。

(1) 提出了 V 形定子动力学解析模型, 揭示了定子的振动特性以及机电耦合特性, 探明了对称和反对模态频率与结构参 数的关系, 发现了驱动足振动位移与两相信号相位差的关系, 探究了对称模态和反对称模态机电耦合系数与变截面尺寸和夹 角的关系。该解析建模方法解决了连接式压电换能器机电耦合系统建模问题，为电机性能预测和控制奠定了基础。

(2) 建立了 V 形直线超声电机动力学模型, 阐明了利用对称模态和反对称模态实现摩擦驱动的机理, 揭示了定、动子之 间的接触动力学行为; 通过电机机械特性预测, 发现了激励频率、电压、相位和负载对电机速度的影响; 通过电机的瞬态特 性分析, 揭示了电压、滑台质量、等效刚度和负载对瞬态特性影响规律; 通过电机电学特性预估, 为电机驱动电路设计以及 电机装配制造质量控制提供了依据。

(3) 基于电机动力学模型, 提出了电机线性化控制方法, 得出了等效驱动力与激励电压和频率的关系, 实现了对电机驱 动的自动调焦系统(最小调焦步距 $1 \mu \mathrm{m}$ )、旋转运动平台(定位误差小于 $0.08^{\circ}$ )和高速机械开关装置(开关时间为 $12 \pm 0.14 \mathrm{~ms}$ )的 有效控制。

(4) 发现了电机输入电流和驱动足振动位移的高次谐波现象, 建立了含有非线性电容的等效电路模型, 分析了其非线性 动力学特性, 提出了新型非线性抑制方法以及驱动控制电路。采用该电路后, 谐波电流总畸变率下降了 $68 \%$, 高次谐波得到 了有效抑制。 